\title{
MATERIAL CHARACTERIZATION OF HIGH DIELECTRIC CONSTANT POLYMER-CERAMIC COMPOSITE FOR EMBEDDED CAPACITOR TO RF APPLICATION
}

\author{
YANG RAO, JIREH YUE and C. P. WONG* \\ School of Materials Science and Engineering, Package Research Center, Georgia Institute of \\ Technology, Atlanta GA 30332-0245
}

(Received 6 November 2001; In final form 3 December 2001)

\begin{abstract}
Embedded capacitor technology can improve electrical performance and reduce assembly cost compared with traditional discrete capacitor technology. Polymer-ceramic composites have been of great interest as embedded capacitor material because they combine the processability of polymers with the desired electrical properties of ceramics. A novel nano-structure polymer-ceramic composite with very high dielectric constant $\left(\varepsilon_{r} \sim 150\right.$, a new record for the highest reported $\varepsilon_{r}$ value of nano-composite) has been developed in our previous work. RF application of embedded capacitors requires that insulating material have high dielectric constant at high frequency $(\mathrm{GHz})$, low leakage current, high breakdown voltage and high reliability. A set of electrical tests have been conducted in this work to characterize the properties of the in house developed novel high dielectric constant polymer-ceramic nano-composite. Results show that this material has faily high dielectric constant in the RF range, low electrical leakage and high breakdown voltage. 85/85 TH aging test has been performed and it had shown this novel high $\mathrm{K}$ material has good reliability. An embedded capacitor prototype with capacitance density of $35 \mathrm{nF} / \mathrm{cm}^{2}$ has been manufactured using this nano-composite with spinning coating technology. This novel nano-composite can be used for the integral capacitors in the RF applications.
\end{abstract}

Keywords: Embedded capacitor; Integral passives; Polymer-ceramic nano-composite; Breakdown voltage; Leakage current; High dielectric constant; RF frequency

\section{INTRODUCTION}

The fundamental building components for all electronic packaging systems consist of active and passive components on an interconnecting substrate [1]. Resistors, inductors, and capacitors are examples of passive components, which represent a class of electronic components that result in no power gain to an electronic application. For example, in current cellular phone applications, the ratio of passive components to active components is nearly 20:1, and nearly $80 \%$ of circuit board area is occupied by discrete passive components. Conventional discrete components have to be mounted onto a printed wiring board (PWB) or interconnected substrate thereby resulting in higher parasitics, lower reliability, and

\footnotetext{
* Corresponding author. Tel.: 404-894-8391; Fax.: 404-894-9140; E-mail: cp.wong@mse.gatech.edu
} 
large attachment area requirements. Discrete capacitors are used in many applications such as noise suppression, filtering, tuning, decoupling, bypassing, termination, and frequency determination, but they occupy substantial geometric surface area, therefore limitations exist in which capacitors can be placed around the chip periphery.

Integral passives are defined as functional elements either embedded in or incorporated on the surface of an interconnecting substrate. With increased production emphasis towards efficient electronic packaging, integral embedded passive technology may satisfy such demands. The main advantages of embedded passive components include: (1) no separate interconnects to the substrate, (2) improved electrical performance, (3) lower cost and (4) ease of processing. Due to increased product demands of increased silicon efficiency, package miniaturization, and higher reliability integral embedded technology will be replacing discrete electrical components.

Embedded passive component technology is being researched by Georgia Tech's Packaging Research Center in conjunction with the novel concept of a System-on-Package (SOP), as shown in the Figure 1 [1]. SOP utilizes a large organic substrate of which multiple layers contain embedded capacitors, resistors, and inductors, respectively. The layers will function together through vias that interconnect the component layers and therefore make them functional.

Since organic PWB will be used as the substrate for the embedded passive, processing parameters of all related components is of utmost importance. Processing conditions for materials systems used with organic substrate are very limited due to the low temperature tolerance of PWB. The maximum temperature of PWB before thermal degradation begins is approximately $250^{\circ} \mathrm{C}$. In order to fabricate a high dielectric MCM-L thin film as well as meet pre-existing material specification, two materials components: polymeric matrix and ceramic filler are used. The polymeric matrix (epoxy) possesses low processing temperatures applicable for PWB applications, but has a low dielectric constant value of around 3 to 4 . The ceramic fillers (Barium Titanate and Lead magnesium niobate-lead titanate (PMN-PT) inherently have a high dielectric constant value of 3000 and 19,316 respectively, making it suitable for decoupling applications. Therefore a hybrid composite will utilize the

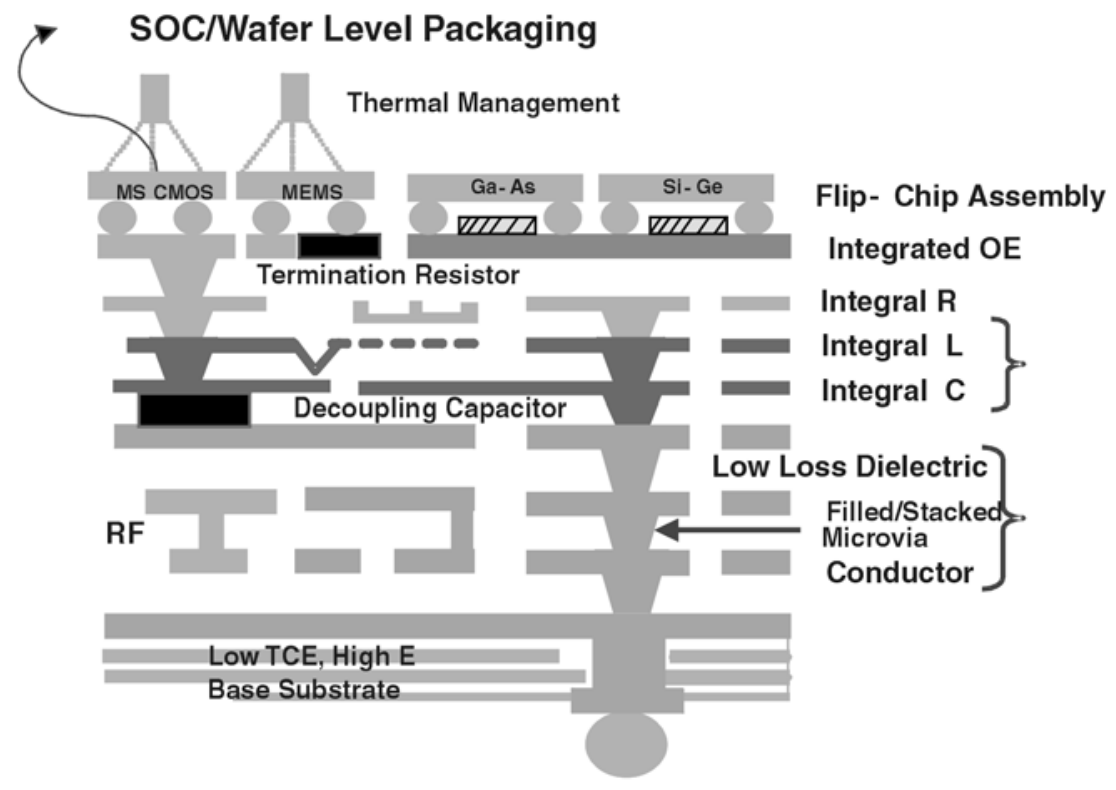

FIGURE 1 Schematic illustration of the SOP substrate. 
processability of the organic polymer matrix and the high dielectric properties of the ceramic fillers. Rao et al. obtained the $70 \%$ ceramic volume loading, resulting in a dielectric constant value of 110 [2]. Such processing method is compatible with MCM-L technology and easily processed resulting in associated low costs. In addition a metal chelating agent, metal acetylacetonate (metal acac), will be used to further increase the dielectric constant of the epoxy material. Upon degradation of the metal acac, charges are released into the polymer matrix thereby resulting in polarization behavior and high dielectric constant property.

The embedded capacitors will be fabricated into a parallel plate configuration, where the capacitance is calculated using the following equation:

$$
C=\frac{\varepsilon_{o} \varepsilon_{r} A}{t}
$$

where $C$ is the capacitance (farads), $\varepsilon_{o}$ is the relative permittivity $\left(8.854 \times 10^{-12} \mathrm{~F} / \mathrm{m}\right), \varepsilon_{r}$ is the dielectric constant, $A$ is the electrode area, $t$ is the thickness of the dielectric medium.

According to Rector, one of the many challenges and difficulties encountered with integral capacitors is low breakdown voltage, high leakage current and poor simultaneous switching noise (SSN) [3]. The power supply noise due to large numbers of simultaneously switching circuit elements has been a concern for a long time. The round trip time-of-flight delay (series inductance) increases as the decoupling capacitor is placed away from the chip [4]. A decoupling capacitor can be used to minimize the on-chip noise or simultaneous switching noise by being directly placed close to the chip, thereby minimizing the interconnect distance. Breakdown voltage is the voltage value at which the material can no longer support the dc voltage passing through the material medium. The leakage current is defined as the dc current per unit area passing through the material under certain dc bias [5].

\section{EXPERIMENTAL}

From the previous work [2], a high $K$ epoxy system was developed by using a Bispheol-A epoxy resin (DER 661 from Dow Chem.) and 5 wt.\% Co(III) acac's as curing catalyst. The reported dielectric constant of this high $K$ epoxy was 6.4 , which has $80 \%$ increase compared to the dielectric constant value of DER661 with the normal curing catalyst. Using this high $K$ epoxy and the combination of two ceramic fillers: lead magnesium niobate-lead titanate (PMN-PT, from TAM ceramics) and $\mathrm{BaTiO}_{3}$ (BT-16 from Cabot Inc.), polymer-ceramic composites were developed by ball milling process. The average particle radius of PMN-PT and $\mathrm{BaTiO}_{3}$ are $0.9 \mu \mathrm{m}$ and $0.050 \mu \mathrm{m}$, respectively. The volume ratio of $\mathrm{PMN}-\mathrm{PT}$ and $\mathrm{BaTiO}_{3}$ was chosen as 4:1 in order to obtain high packing density. In order to achieve good dispersion of the ceramic fillers in epoxy matrix, a phosphate ester (Byk-w 9010, Dow Chem.) was used as the surfactant. A sample (composite-I) containing 85 vol.\% filler loading were ball milled for approximately 1 day at a speed of $220 \mathrm{r} / \mathrm{min}$ to obtain good particle dispersion [6]. The viscosity of the sample was adjusted by the addition of solvents (NMP). In order to obtain the dielectric constant values for the epoxy system, prototype of embedded capacitors were fabricated (see Figure 2). Capacitance measurements were taken using a HP 4263A LCR meter at $10 \mathrm{kHz}$. Dielectric constant values were calculated from the capacitance data using Eq. 1. The dielectric constant of the composite-I was calculated as 150 .

A set of electrical tests was conducted to characterize the high $K$ epoxy-ceramic nano-composite. First of all, dielectric constant was measured in the frequency range from $10 \mathrm{kHz}$ to $1.8 \mathrm{GHz}$ by using a HP 4291A RF Impedance/Material Analyzer. Secondly, the leakage current was measured using a amperemeter by connecting the sample with two electric microprobes. Thirdly, an adjustable dc power supply was used to measure the breakdown 


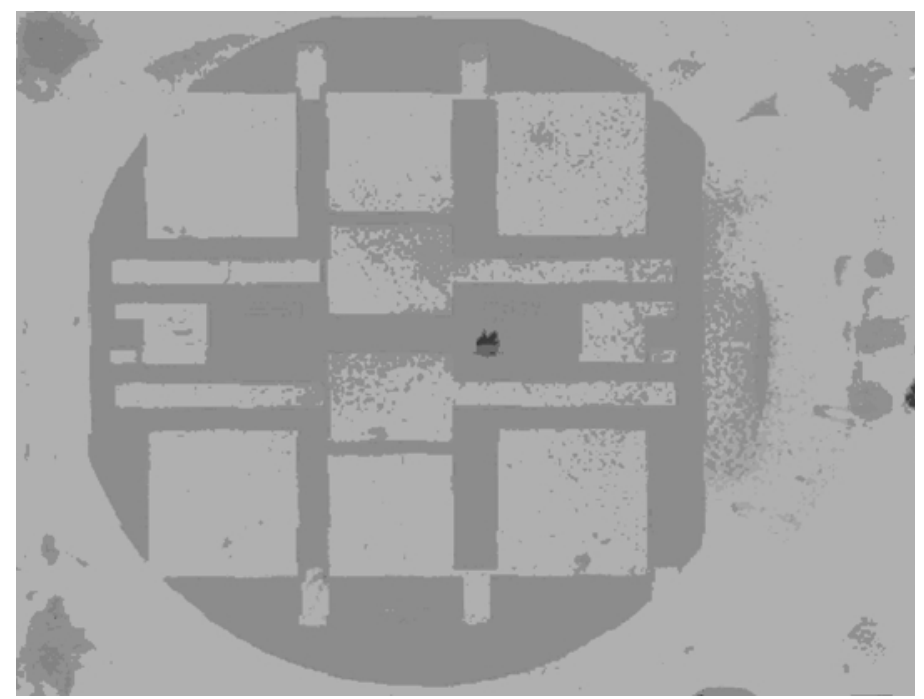

FIGURE 3 The dielectric constant of composite-I at different frequency.

voltage of the composite. Fourthly, a programmable hot plate was used with the HP 4263A LCR meter to characterize the thermal tolerance of a typical capacitor, with the thickness of $3.75 \mu \mathrm{m}$. The thermal tolerance is defined as the relationship between the capacitance and the temperature. Finally, capacitance was measured for the same capacitor after 85/85 thermal humidity $(\mathrm{TH})$ aging test.

\section{RESULTS AND DISCUSSION}

Figure 3 shows the dielectric constant of the composite at different frequency. It was found that the decrease of the dielectric constant was less than $10 \%$ from $10 \mathrm{kHz}$ to $1.8 \mathrm{GHz}$. This

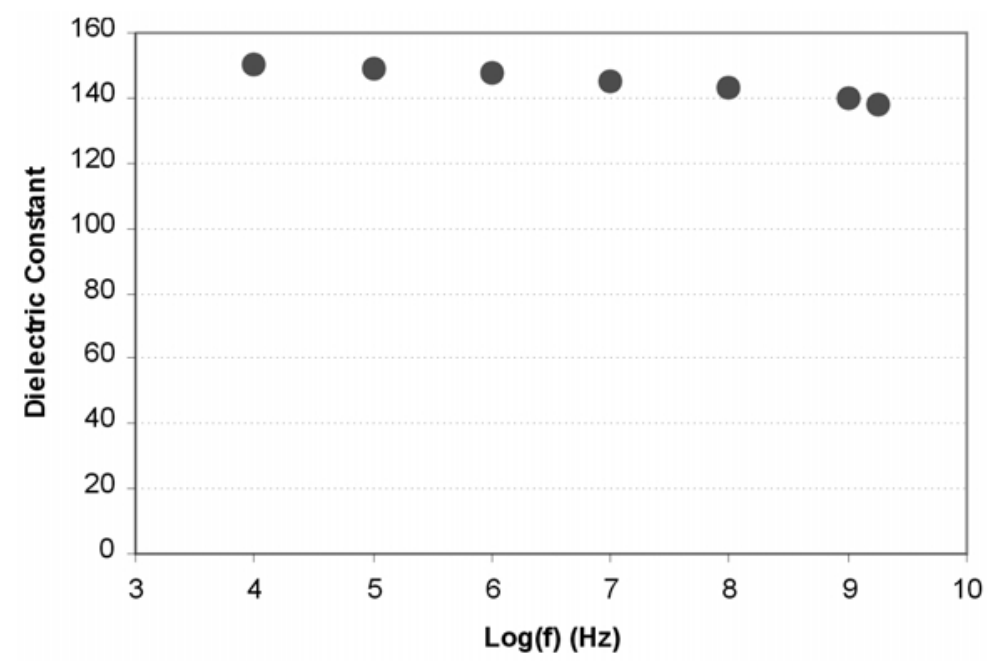

FIGURE 2 Prototype of capacitors fabricated by thick film technology. 


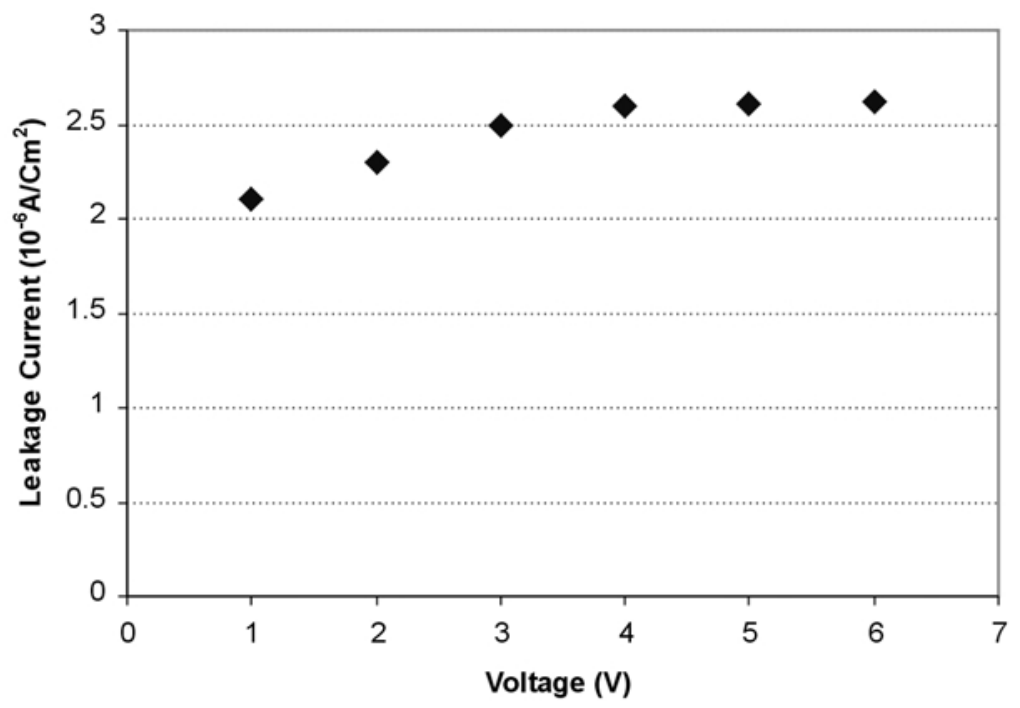

FIGURE 4 The leakage current of the composite-I.

outstanding feature of low loss makes the composite-I suitable for making the embedded decoupling capacitor for RF application.

Figure 4 shows the leakage current of the composite-I with the thickness of the material is $3.75 \mu \mathrm{m}$. The leakage current is less than $2.6 \mu \mathrm{A} / \mathrm{cm}^{2}$ under $6 \mathrm{~V}$ dc bias, and it will level off at higher dc bias. The leakage current of composite-I can fulfill the requirement of the embedded capacitor application.

Figure 5 shows the capacitance density change of an embedded capacitor prototype using composite-I as insulating material at different temperature. Basically, the dielectric constant

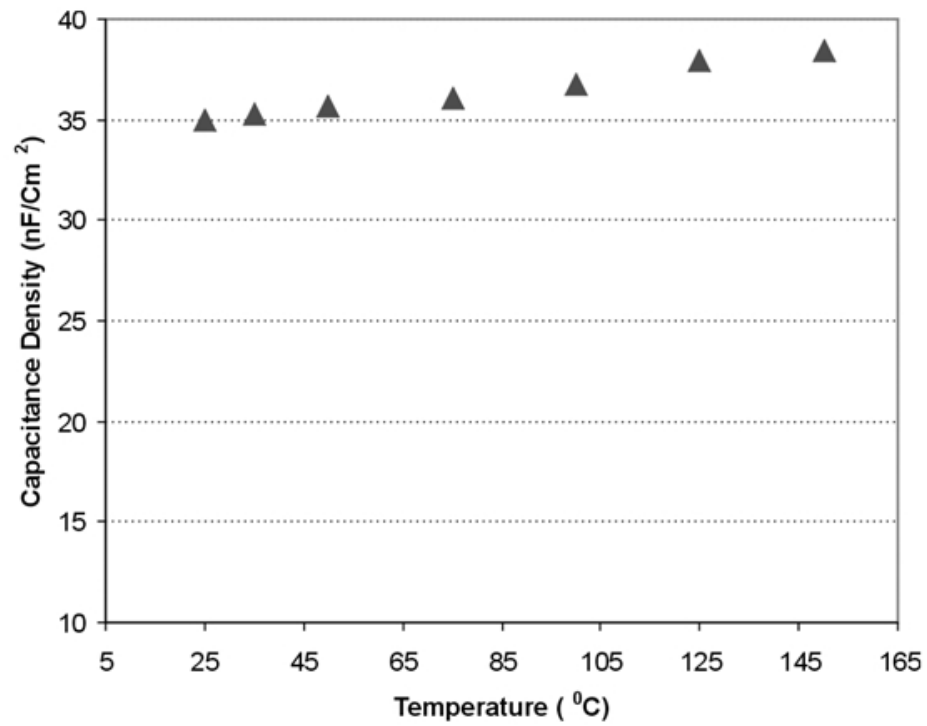

FIGURE 5 The capacitance density of composite-I with $3.75 \mu \mathrm{m}$ thickness at different temperature. 


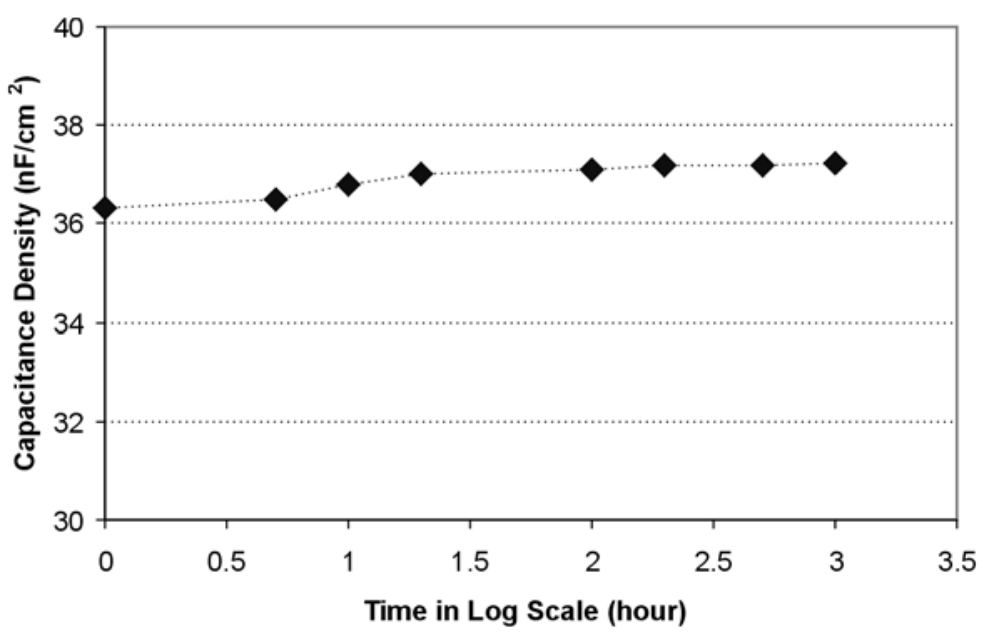

FIGURE 6 The capacitance density of composite-I with $3.75 \mu \mathrm{m}$ thickness after $85 / 85 \mathrm{TH}$ aging test.

increases from $35 \mathrm{nF} / \mathrm{cm}^{2}$ to $38.4 \mathrm{nF} / \mathrm{cm}^{2}$ when temperature changes from $25^{\circ} \mathrm{C}$ to $155^{\circ} \mathrm{C}$, which is an increase less than $10 \%$. However, it is hard to obtain the dielectric constant change according to thermal loading, since it is difficult to exclude the geometry change of the capacitor.

Figure 6 shows the capacitance density of the previous capacitor after certain time at 85/85 $\mathrm{TH}$ aging test. It was found that the capacitance increased in the first 24 hours of the $85 / 85$ TH aging test, after that, capacitance did not change much. It is believed that moisture absorption plays an important role for the initial capacitance increase. The moisture absorption becomes saturated, after 24 hours. Figure 7 shows the moisture absorption of the composite-I under $85 / 85$ condition, which proves the assumption. Because the total moisture

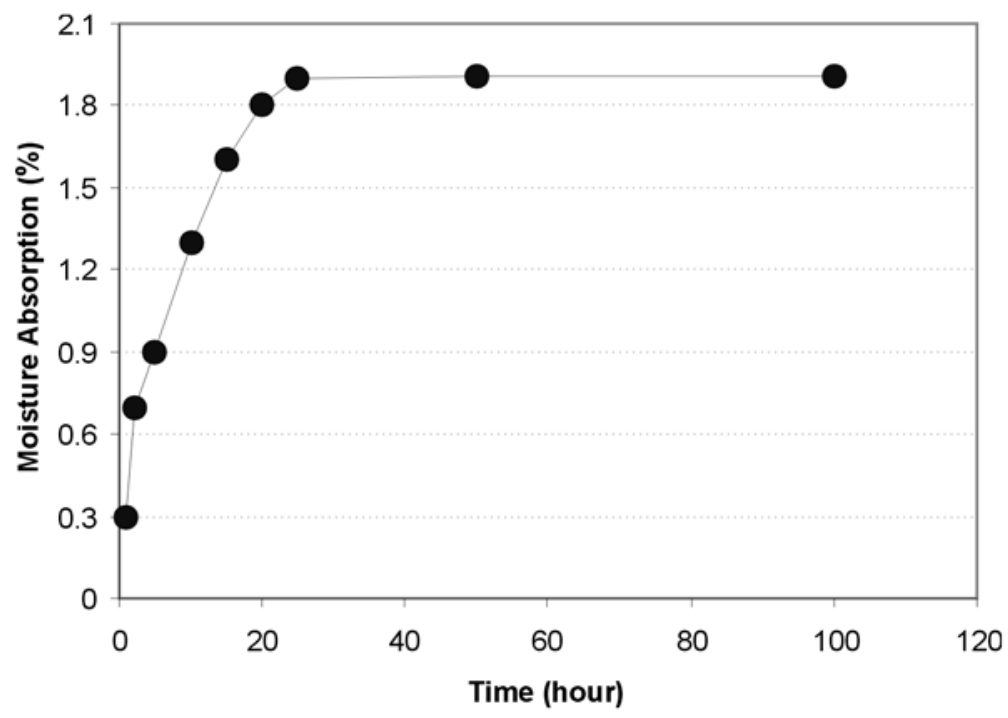

FIGURE 7 The moisture absorption of composite-I with $3.75 \mu \mathrm{m}$ thickness at $85 / 85 \mathrm{TH}$ condition. 
absorption is less than $2 \%$, the capacitance density increase $2.5 \%$ after $1000 \mathrm{hr}$ at $85 / 85 \mathrm{TH}$ aging test. There is no electrical failure happened during the $85 / 85 \mathrm{TH}$ aging test.

The breakdown voltage of the composite-I is measured higher than $1.7 \times 10^{7} \mathrm{~V} / \mathrm{m}$, which is high enough as the insulating material for embedded capacitor [3].

\section{CONCLUSIONS}

Using the on house developed high $K$ epoxy as matrix, composite-I with dielectric constant equal to 150 was developed at 85 vol.\% ceramic loading. A set of electrical and reliability tests have been conducted to characterize the physical properties of the composite-I. It was found that composite-I has stable dielectric constant in the wide frequency range $(10 \mathrm{kHz}-1.8 \mathrm{GHz})$, very high breakdown voltage and small leakage current. The embedded capacitor prototype was fabricated using composite-I and achieved the capacitance density of $35 \mathrm{nF} / \mathrm{cm}^{2}$. The capacitance density change of this capacitor is less than $10 \%$ from $25^{\circ} \mathrm{C}$ to $155^{\circ} \mathrm{C}$. In addition, composite-I has relatively small moisture absorption $(<2 \%)$, which leads to a stable capacitance under $85 / 85 \mathrm{TH}$ aging test. There is no electric failure happened after $1000 \mathrm{hr} 85 / 85 \mathrm{TH}$ aging test. As such, composite-I can be a very good material candidate for embedded capacitor in the RF application.

\section{Acknowledgement}

The authors gratefully acknowledge NSF-PRC and Draper Lab for supporting this work. The authors also would like to thank TAM Ceramics for donating the ceramic materials.

\section{References}

[1] Tummala, R. R., Chahal, P. and Bhattacharya, S. (1998). Recent advances in integral passives at PRC. IMAPS 35th Nordic Conference, Sweden, September 1998.

[2] Rao, Y., Ogitani, S., Kohl, P. and Wong, C. P. (2000). Novel high dielectric constant nano-structure polymerceramic composite for embedded capacitor application. Proceeding of ECTC 50th, Las Vegas, May 2000.

[3] Ramesh, S., Haung, C., Liang, S. and Giannelis, E. (1999). Integrate thin film capacitors: Interfacial control and implications on fabrication and performance, Electronic Components and Technology Conference, 1999.

[4] Tummala, R., Rymaszewski, E. J. and Klopfenstein, A. G. (1997). Microelectronics Packaging Handbook, 2nd edn. International Thompson Publishing, New York.

[5] Grob, B. Basic Electronics, 6th edn. McGraw-Hill Book Company, New York.

[6] Ogitani, S., Bidstrup-Allen, S. A. and Kohl, P. (2000) IPC Conference, San Diego, CA, April 2-6, 2000.

[7] Ogitani, S., Bidstrup-Allen, S. A. and Kohl, P. (1998). In: Proc. 23rd International Electronics Manufacturing Technology Symposium, pp. 199-205.

[8] Wong, C. P., Shi, S. H. and Jefferson, G. (1998). High performance no-flow underfills for low-cost flip-chip applications: Material characterization. IEEE Transactions on Components, Packaging, and Manufacturing Technology: Part A, 21(3), 450-458.

[9] Rector, J. (1998). Economic and technical viability of integral passives. IEEE 48th Electronic Components and Technology Conference, 1998, p. 218.

[10] Rector, J., Dougherty, J. and Brown, V. (1997). Integrated and integral passive components: A technology roadmap. Electronic Components and Technology Conference, 1997.

[11] Schaper, L. and Lenihan, T. (1998). Passives go into hiding. Advanced Packaging, pp. 22-26.

[12] Park, J. Y., Bhattacharya, S. K. and Allen, M. G. (1997). Fully integrated passives module for filter applications using low temperature process. ISHM Philadelphia, PA, 1997, pp. 592-597.

[13] Das-Gupta, D. K. and Doughty, K. (1988). Thin Solid Films, 158, 93-105. 

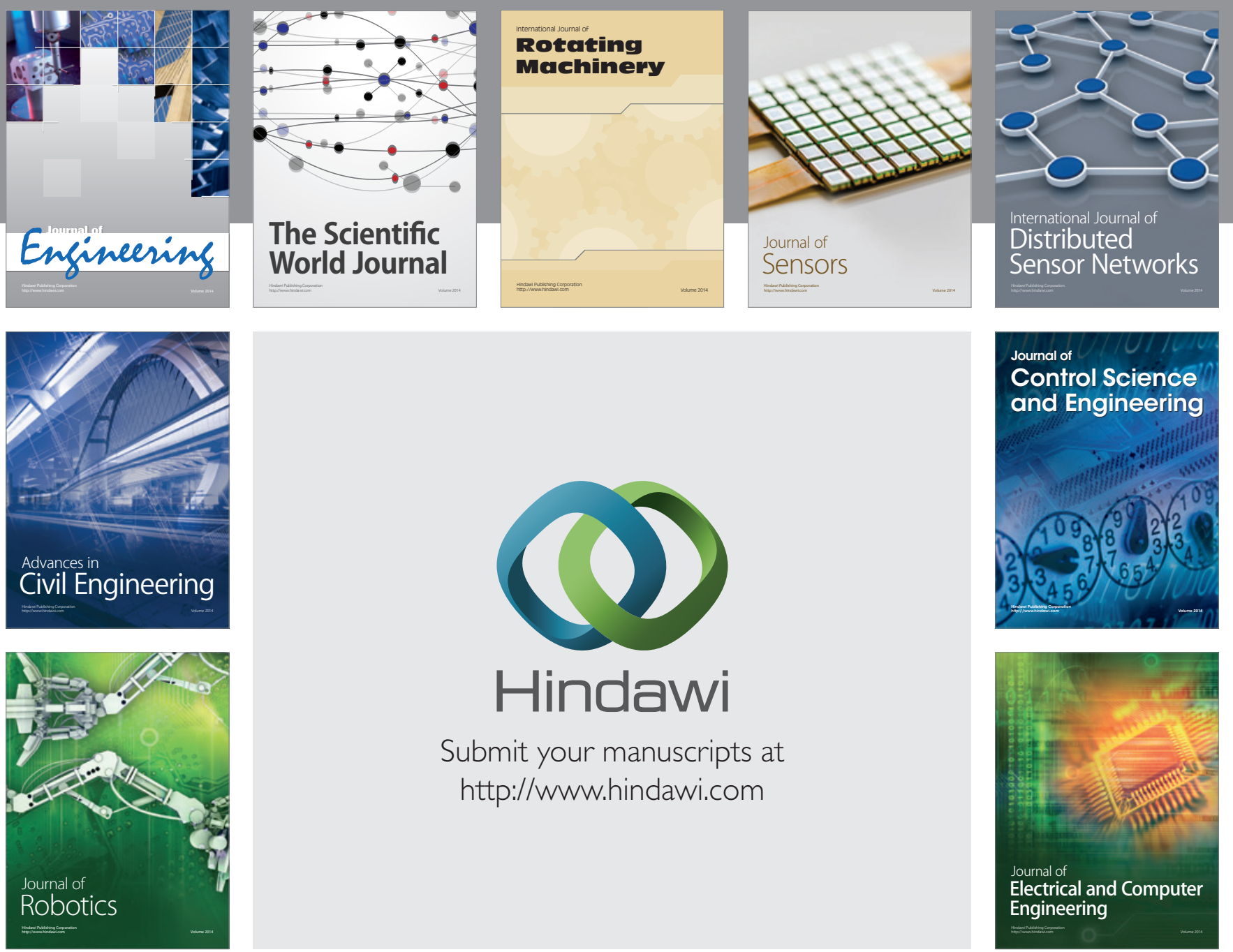

Submit your manuscripts at

http://www.hindawi.com
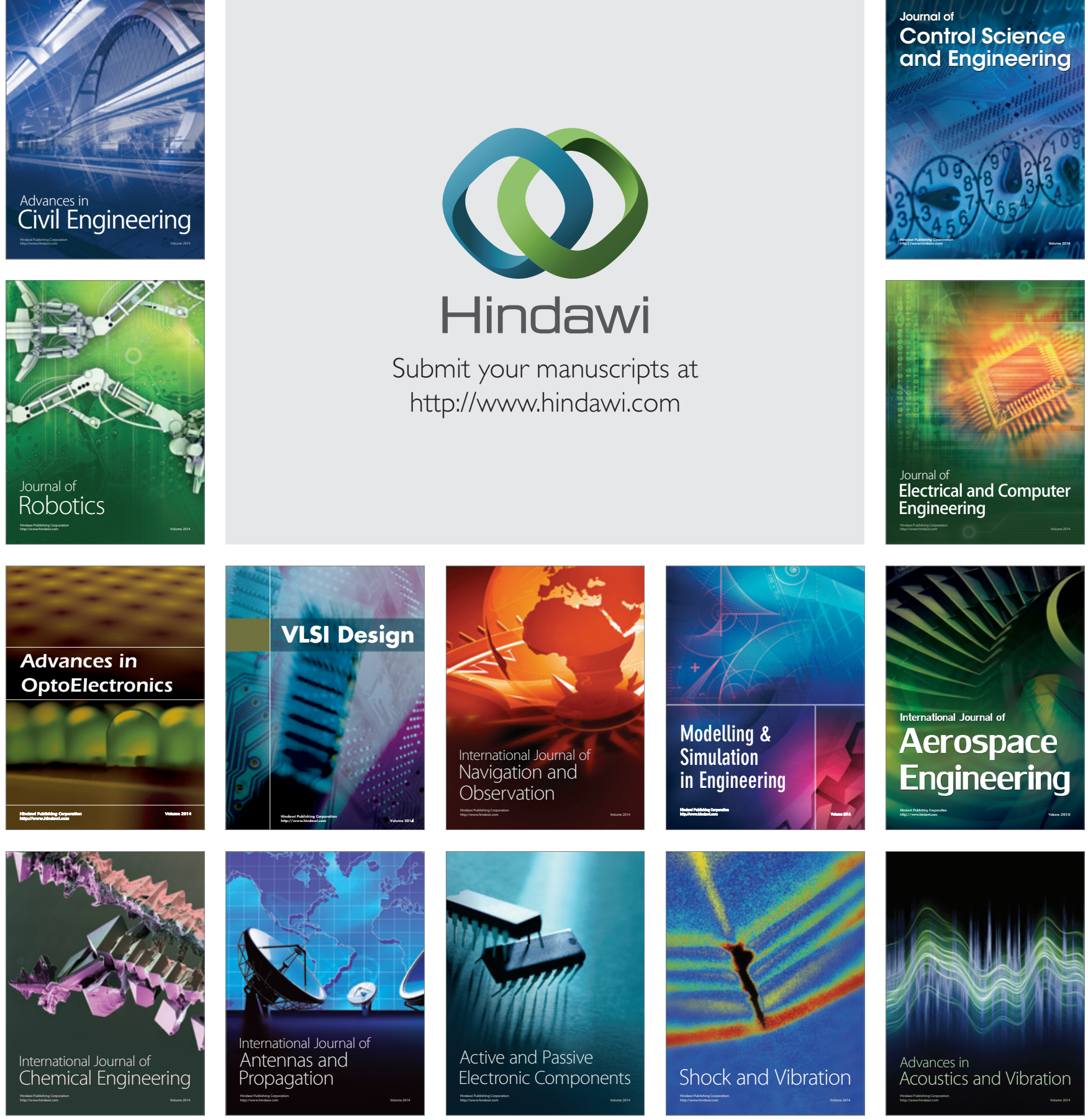